\title{
The flipflop orphan genes are required for limb bud eversion in the Tribolium embryo
}

\author{
Susanne Thümecke', Anke Beermann², Martin Klingler ${ }^{3}$ and Reinhard Schröder ${ }^{2 *}$ (D)
}

\begin{abstract}
Background: Unlike Drosophila but similar to other arthropod and vertebrate embryos, the flour beetle Tribolium castaneum develops everted limb buds during embryogenesis. However, the molecular processes directing the evagination of epithelia are only poorly understood.

Results: Here we show that the newly discovered genes Tc-flipflop1 and Tc-flipflop2 are involved in regulating the directional budding of appendages. RNAi-knockdown of Tc-flipflop results in a variety of phenotypic traits. Most prominently, embryonic limb buds frequently grow inwards rather than out, leading to the development of inverted appendages inside the larval body. Moreover, affected embryos display dorsal closure defects. The Tc-flipflop genes are evolutionarily non-conserved, and their molecular function is not evident. We further found that Tc-RhoGEF2, a highly-conserved gene known to be involved in actomyosin-dependent cell movement and cell shape changes, shows a Tc-flipflop-like RNAi-phenotype.

Conclusions: The similarity of the inverted appendage phenotype in both the flipflop-and the RhoGEF2 RNAi gene knockdown led us to conclude that the Tc-flipflop orphan genes act in a Rho-dependent pathway that is essential for the early morphogenesis of polarised epithelial movements. Our work describes one of the few examples of an orphan gene playing a crucial role in an important developmental process.
\end{abstract}

Keywords: Appendage formation, Epithelial morphogenesis, Evagination, Orphan flipflop gene, PCP, RhoGEF2, Tissue folding, Tribolium castaneum

\section{Background}

The general bauplan of the insect leg is highly conserved in evolution and so are the genes controlling appendage growth and patterning [2]. Yet, principal topological differences within the insects exist. In the fruit fly Drosophila, the leg anlagen invaginate from the larval epidermis and internalise to develop inside the body cavity as imaginal discs. During pupation, appendages evert and only become functional in the adult. In contrast, ventral appendages of the flour beetle Tribolium start as everting epidermal bulges that subsequently grow in length during embryogenesis. This mechanism of appendage formation is representative for most arthropods and similar to apical epidermal ridge formation in vertebrates [59]. Eventually Tribolium larvae hatch with fully differentiated, functional appendages [49].

\footnotetext{
* Correspondence: reinhard.schroeder@uni-rostock.de

¿Universität Tübingen, Auf der Morgenstelle 15, D-72076 Tübingen, Germany Full list of author information is available at the end of the article
}

Bud formation takes place in a restricted area of the epithelium where cells collectively polarise, undergo cell shape changes and, as a consequence, evaginate. Once this crucial decision is made, the bud grows in length and eventually differentiates $[19,54,60]$. The coordinated contractility of a group of cells at their apical or basal cortices provides the cellular basis for this morphogenetic event: apical constriction leads to tissue invagination while basal constriction results in the formation of an external bud. Constriction at one cortex of a cell usually goes along with expansion of the membrane at the opposite side [22].

To date, morphogenetic processes that involve apical constriction are intensely studied in a variety of developmental contexts. Most prominent examples are the infolding of cell sheets during gastrulation or neurulation, blastopore formation, trachea development, dorsaland neural tube closure as well as embryonic tissue sealing during wound healing [22, 25, 27, 34, 40, 45]. 
However, tissue eversion as a consequence of basal constriction is less well understood and has been analysed in only a few cases: the formation of the midbrainhindbrain boundary constriction and morphogenesis of the optic-cup in vertebrates, and notochord formation in an urochordate $[9,14,28,29,33]$. Classical studies in the polyp Hydra describe basally constricted cells within epithelial sheet curvature during reproductive bud initiation $[12,58]$.

Different cellular mechanisms such as differential growth or compressing forces from neighbouring cells have also been shown to initiate tissue bending and have been described for morphogenetic events like branching of developing epithelia or gut looping [56]. Moreover, all the described processes are likely to synergise with other types of cell behaviour, such as directed cell migration into the region where a bud will form, or changes in adhesive properties once a bud protrudes out of the plane of an epithelium.

In any case, epithelial cell shape changes require the dynamic and spatial reorganisation of the actomyosin network. Its assembly and disassembly is controlled by small GTPases like RhoA (ras homologue family member A). RhoA becomes activated by the guanine nucleotide exchange factor RhoGEF2 which is transported to the apical cell cortex along the polarised microtubule network through association with the plus-end binding protein EB1 at the tips of the growing microtubules. At the apical cortex, active RhoA triggers myosin contraction through the Rho-associated coiled-coil kinase (ROCK) [27, 41].

Rho family GTPases, the effectors of myosin constriction, are also a target of the planar cell polarity (PCP) signalling pathway [30] that coordinates the behaviour of cells within an epithelium. The aligning of activated myosin through PCP along an axis eventually leads to polarised tissue-bending and -folding in one direction exemplarily seen during neural tube folding [35]. PCP involves the non-canonical Wnt-signaling pathway upstream of Rho [6].

In addition, the Rho/RhoGEF2/ROCK cassette is employed by other upstream signals and factors like the Jun-N-terminale-kinase (JNK-) or G-protein-coupled receptors (GPCR) [17, 21, 37].

In order to further understand the crucial aspects of early evagination processes we analysed genes in Tribolium resulting in a hitherto not described larval knockdown phenotype of inverted rather than everted larval appendages.

Here, we focus on the novel Tc-flipflop1 (Tc-ff1) gene that was identified in the genome-wide RNAi (RNA interference) screen iBeetle [48] and a newly identified flipflop paralogue (Tc-ff2). RNAi-based knockdown of Tc-ff results in the formation of inverted but otherwise fully developed legs inside the larval thorax rather than growing out distally. A similar appendage phenotype was observed in an insertional mutant identified in the GEKU screen [55] which is located in the RhoGEF2 gene. Furthermore, we found that both Tc-flipflop genes as well as Tc-RhoGEF2 are essential for the integrity of morphogenetic movements of embryonic cells and extraembryonic membranes. We propose that in the limb-field, the very early decision of an epithelium to either invaginate or evaginate depends on Rho associated signalling with the novel $T c-f f$ genes as essential mediators to secure tissue eversion. Whether restricted only to Tribolium or fast evolving yet present in other animals, the Tc-ff orphan genes highlight the involvement/importance of novel factors in early epithelial morphogenesis and appendage formation.

\section{Methods}

Animal stocks

Beetle adults and embryos (Tribolium castaneum, nGFP line) [43] were kept under standard conditions on wheat flour at $30{ }^{\circ} \mathrm{C}$ [5] and used for parental RNAi, in situ hybridisation and live imaging.

\section{RNAi mediated knockdown}

For gene specific knockdown non-overlapping fragments were ordered from Eupheria Biotech GmbH $(1 \mu \mathrm{g} / \mu \mathrm{l}$, $3 \mu \mathrm{g} / \mu \mathrm{l})$. For parental RNAi young adult females were sedated on ice and fixed on a petri dish using doublesided adhesive tape. dsRNA (Tc-ff, $500 \mathrm{ng} / \mu \mathrm{l} ; T c-R h o-$ GEF2, $200 \mathrm{ng} / \mu \mathrm{l})$ was injected into the abdomen under a stereomicroscope using a glass capillary connected to a manually controlled syringe. Gene specific effects for all results shown were validated with at least two nonoverlapping fragments (NOFs) for each gene (Tc-ff1: NOF1 basepairs 1-320 (xx-90,314-2), NOF2 bp 340-659 (xx-90,314-1), Tc-ff2: NOF1 bp 129-416 (xx-90,313-3), NOF2 bp 417-710 (xx-90,313-2), Tc-RhoGEF2 NOF1 bp 2338-2664 (iB_03492), NOF2 bp 7408-7907) (iB_00510)). Eggs were collected and either fixed (in situ hybridisation, antibody staining, morphological analysis) or incubated at $30{ }^{\circ} \mathrm{C}$ to develop a cuticle. $T c-D l l$ dsRNA was injected as a control to validate gene-specific effects.

\section{Molecular biology and expression analysis}

For whole mount in situ hybridisation gene-specific primers were used (Metabion) to amplify gene fragments via PCR using cDNA synthesised from total RNA. The amplified fragments were subcloned into the pCR4 vector (TOPO-TA Cloning Kit, Invitrogen). In vitro transcription for synthesis of DIG-labelled RNA probes was performed using the DIG RNA Labelling Kit (Roche Applied Science). Whole mount in situ hybridisation was performed as previously described [46]. Staining was 
achieved through application of an Anti-Digoxigenin-AP antibody in combination with NBT/BCIP (Roche Applied Science).

Antibody staining was carried out as previously described [51] using antibodies raised against short peptides corresponding to Flipflop1 (NH2-CPKTTKPKAKCONH2), Flipflop2 (NH2-CSKNTEHKTK-CONH2) (Pineda Antibody-Service) and Cleaved Dcp-1 (\#9578, Cell Signaling Technology), respectively. A Biotin-SPconjugated AffiniPure Anti-Rabbit lgG antibody was used as secondary antibody (Jackson ImmunoResearch Europe Ltd). Staining was carried out using Vectastain ABC-AP (Vector Laboratories) and NBT/BCIP.

\section{Live imaging}

Eggs (nGFP) were dechorionated in diluted bleach, washed, positioned on a microscope slide and covered with halocarbon oil (Voltalef 10S). Injections were performed under an inverted microscope using a micromanipulator. Live imaging was carried out using a Zeiss Z.1 microscope (Zen 2.3 software) equipped with a motorised stage. Pictures were taken every 1-5 min. Images and videos were processed with Adobe Photoshop CS5.

\section{Databases}

\section{Genomes:}

Tribolium castaneum: http://bioinf.uni-greifswald.de/ gb2/gbrowse/tcas4/

UCSC Genome Browser: https:/genome-euro.ucs c.edu/cgi-bin/hgHubConnect? redirect=manual\&source =genome.ucsc.edu

\section{RNAi induced phenotypes:}

iBeetle-Base: http://ibeetle-base.uni-goettingen.de [10].

\section{Protein structure:}

HHpred: https://toolkit.tuebingen.mpg.de/\#/tools/hhpred [52].

SMART: http://smart.embl-heidelberg.de/smart/set_mode.cgi? NORMAL=1 [50].

\section{Results}

The novel gene Tc-flipflop reveals a new RNAi knockdown phenotype

In wildtype Tribolium larvae, appendages develop as everted structures outside the larval body (Fig. 1a). The RNAi mediated knockdown of the gene Tc-flipflop1 (Tcff1) results in an enigmatic novel RNAi cuticle phenotype where larval appendages developed inside the larval body (outside-in phenotype). This gene was uncovered during the iBeetle prescreen when randomly selected cDNAs were tested for their function [48]. In $T c-f f^{\text {RNAi }}$ cuticles, various numbers of head - or thoracic appendages appear to be missing or shortened when focussing onto the cuticle surface (Fig. 1b, circles). However, those appendages are indeed present inside the larva as seen in optical sections (Fig. 1b'). These inverted structures do not show any obvious developmental defects, revealing distal segments (flagellum, pretarsal claw) oriented towards the lumen, while podomers and joints seem fully differentiated (Fig. 2a, b).

\section{There are two Tc-flipflop genes in the Tribolium genome}

In addition to the original Tc-flipflop gene, we identified an obvious Tc-ff paralog in the Tribolium genome. Both genes show $47 \%$ sequence similarity at the amino acid level and were therefore named Tc-ff1 (TC032552) and Tcff2 (TC030881), respectively. Tc-ff1 and Tc-ff2 are short genes (738 and $756 \mathrm{bp}$ ) with two exons each (Fig. 1c, d) that lack any conserved domains.

ff1 is also present in other Tribolium species including T. confusum, T. madens and T. freemani. Due to their incomplete annotation, it is not yet clear whether those genomes all contain a true ff2 paralogue (UCSC Genome Browser data, not shown). However, the genes cannot be found in any other sequenced genomes, including more distantly related coleopteran species.

In Tribolium castaneum, both Tc-ff genes are ubiquitously expressed in all embryonic stages at mRNA level (Additional file 1: Fig. S1B-E, F, H). The initially observed outside-in appendage phenotype was validated for both genes using two independent, non-overlapping fragments (NOFs) (Fig. 1c, d).

\section{The "flipflop syndrome"}

RNAi mediated knockdown of both Tc-ff genes results in the outside-in appendage phenotype but also reveals additional lesions of the larval cuticle such as the invagination of abdominal segments and the failure of dorsal closure. These alterations from the wildtype collectively comprise the "flipflop syndrome".

In addition to the phenotype of inwards grown head appendages and legs, the invagination phenotype either included posterior abdominal segments (ff1, 16\%, $n=205$; $f f 2,19 \%, n=113$ ) (Fig. 3e) or just the appendages of the last abdominal segment (urogomphi) (ff1, 16\%, $n=205$; ff2, $17 \% n=113$ ). Only stronger affected cuticles display an incomplete closure of the dorsal epidermis (ff1, 19\%, $n=205$; $f f 2,20 \%, n=113$ ) (Fig. 3c, h). Not analysable cuticle remnants $(f f 1,14 \%, n=205$; ff2, 5\%, $n=113$ ) (Fig. 3f) and the complete failure of cuticle formation ("empty egg") (ff1, 25\%, $N=443$; ff 2, 55\%, $N=322$ ) represented the strongest detectable knockdown effects. RNAi experiments for either $T c-f f 1$ or $T c-f f 2$ displayed the whole range of the "flipflop syndrome" but differed in penetrance being higher in Tc-ff2 RNAi compared to Tc-ff1 (Fig. 4). The phenotype of inwards grown head appendages and legs was observed more often in Tc-ff2 RNAi experiments $(67 \%, n=113)$ when compared to Tc-ff1 (ff1, 40\%, $n=205)$ (Fig. 4). The 


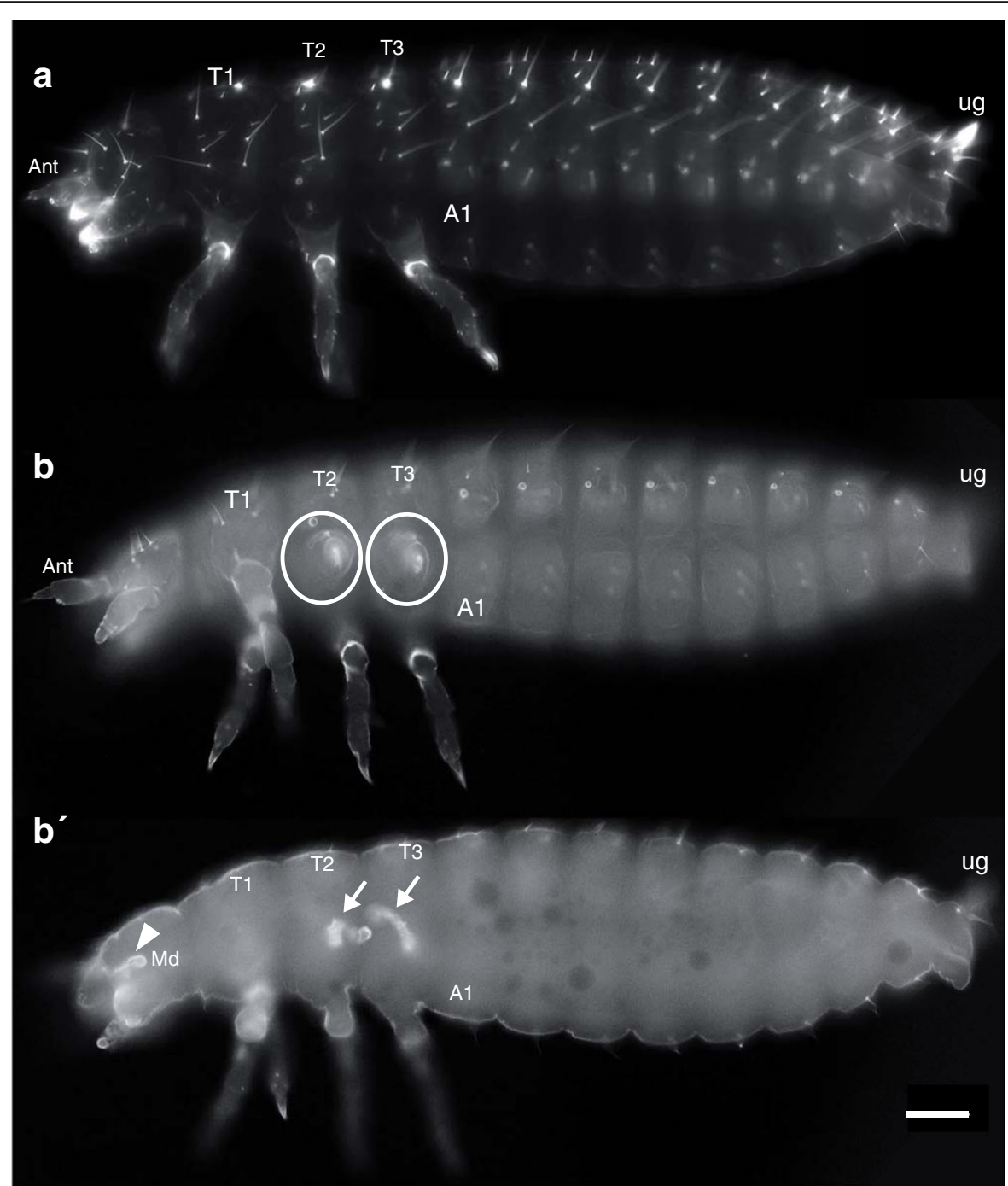

C

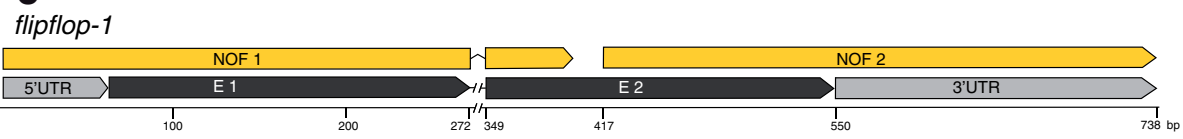

d

flipflop-2

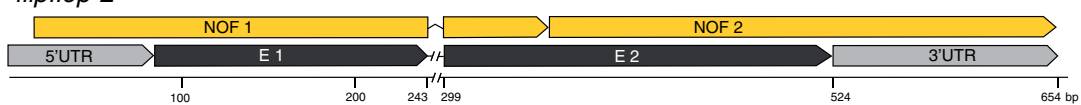

Fig. 1 Larval Tc-flipflop ${ }^{\text {RNAi }}$ phenotype. (a) Wildtype cuticle. All appendages develop as everted structures. Each thoracic segment bears one pair of visible legs. (b-b') Tc-ff ${ }^{\text {RNAi }}$ phenotype. (b) Surface view, (b') optical section. (b) In the thoracic segments 2 and 3, one leg is everted normally. Of the other legs, only the coxa is visible as an outer structure (circle) while the remaining leg is internalised (b', arrows). (c, d) Gene organisation of Tc-ff1 (c) and Tc-ff2 (d) genes including NOF (non-overlapping-fragment) positions used for RNAi experiments. A1 abdominal segment 1; Ant antenna; Md mandible; T1-3 thoracic segment 1-3; ug urogomphi; scale bar (a-b') $100 \mu \mathrm{m}$; all panels in all pictures: anterior to the left

number of inversion events observed varies from only one inverted appendage (see for example Fig. 1b') to as many as 8 and 9 (Fig. 3b-h) or even 10 (not shown) in more extreme cases (Additional file 2: Table S2). Combined RNAi experiments that included double stranded RNA for both genes did not result in stronger or different cuticle phenotypes compared to the single RNAi experiments (Additional file 2: Table S1), nor were "empty egg" phenotypes more frequent. The quantitative analysis was carried out for the first two egg lays of RNAi experiments using a NOF directed at the 3 ' region of the genes (Fig. 1c). We were able to validate the flipflop outside-in phenotype 


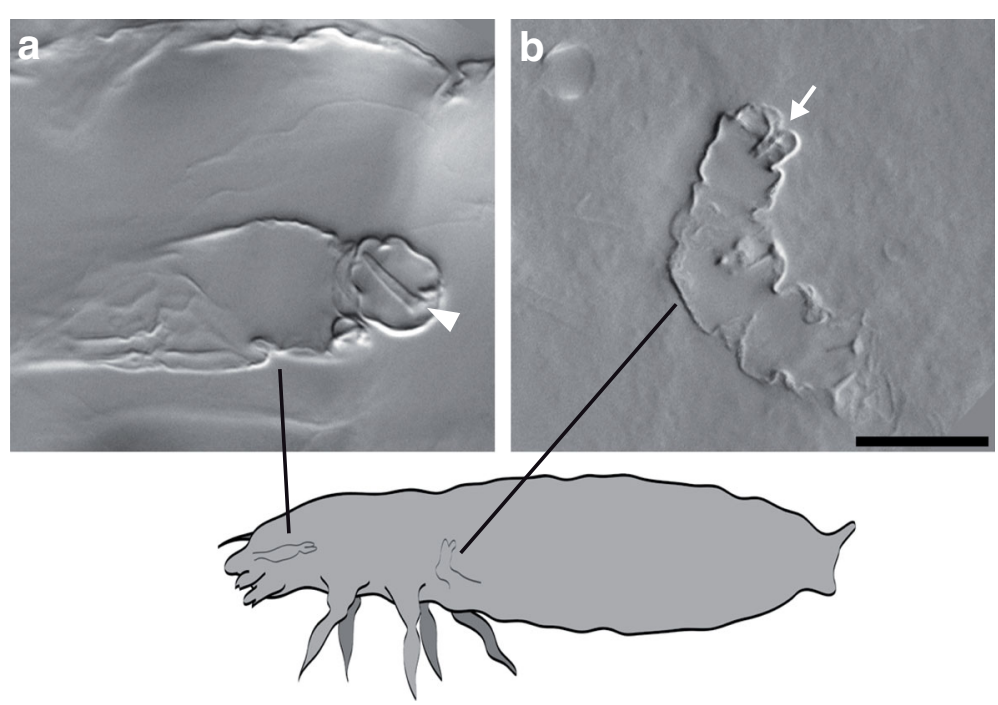

Fig. 2 Detailed view of the larval Tc-flipflop ${ }^{\mathrm{RNAi}}$ phenotype. a, b Magnified view of a larval inverted antenna a and leg $\mathbf{b}$. Internalised parts are fully differentiated; the distalmost structures (antennal flagellum) (arrowhead in a) and the pretarsal claw (arrow in b) develop normally and display the inside-out event. Scalebar $20 \mu \mathrm{m}$

using the $5^{\prime}$ NOF but with a much lower frequency as the majority of embryos did not reach the larval stage ("empty egg"). Using antisense mRNA probes for both genes on $T c-f f^{\text {RNAi }}$ embryos we observed strongly reduced staining compared to untreated wildtype embryos (Additional file 1: Fig. S1F-H), indicating the efficiency of the knockdown.

\section{Tc-flipflop determines the directionality of embryonic appendage formation in Tribolium}

To answer the question of when during development of $T c-f f^{\text {RNAi }}$ embryos the invagination of appendages starts, we analysed the early embryonic morphology as well as the expression profile of the distal leg marker Tc-Distalless $(T c-D l l)[4,8]$ and the segmentally expressed $T c$ -

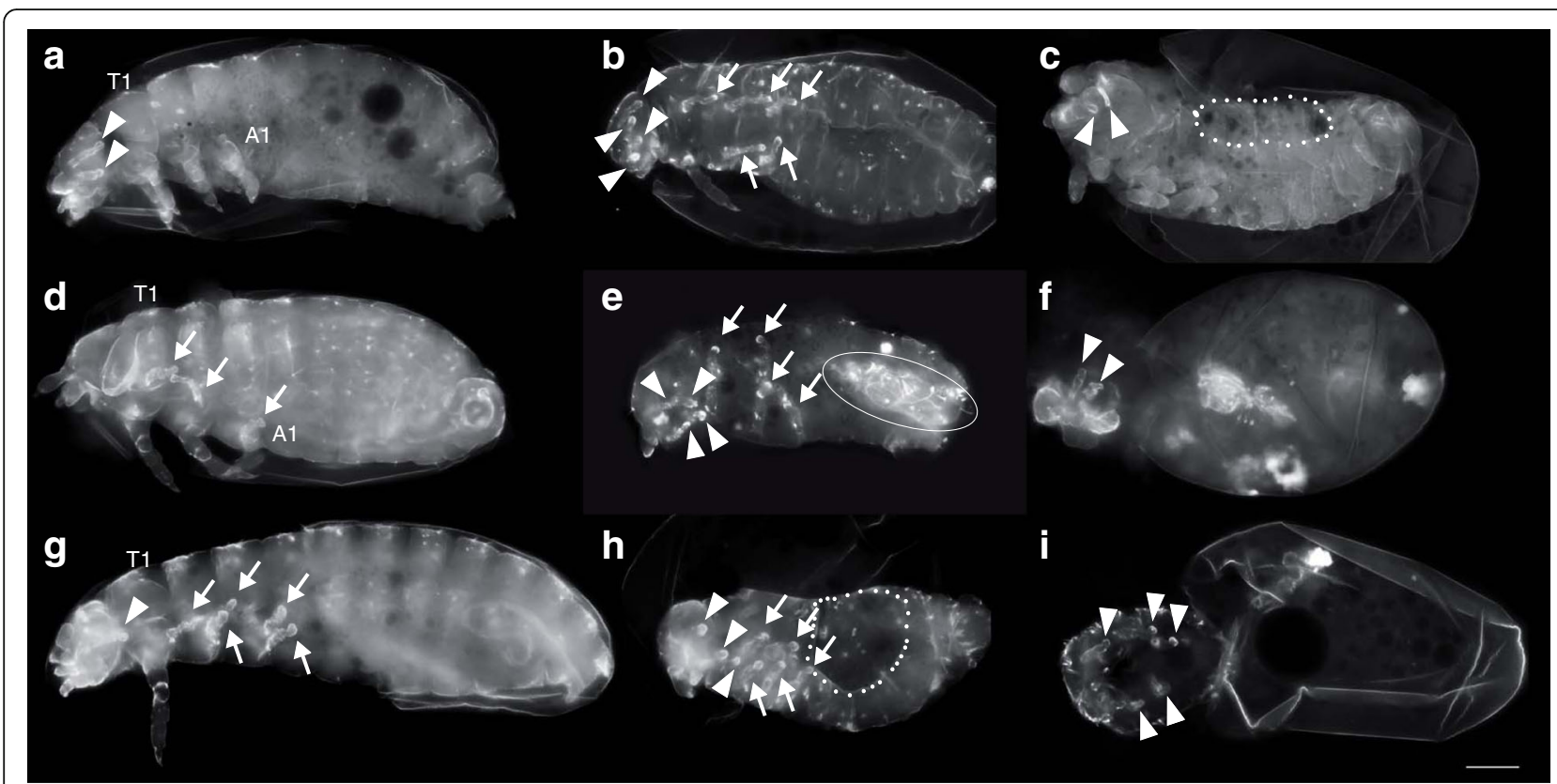

Fig. 3 The "flipflop syndrome". a-i Larval RNAi phenotypes of Tc-ff1 a-c, Tc-ff2 $\mathbf{d}-\mathbf{f}$ and Tc-ff1/ff2 $\mathbf{g}$-i parental double-knockdown. a-i The enigmatic phenotype of inverted head appendages (arrowhead) and legs (arrows) in different combinations. In addition, inverted abdominal segments (e, circle) and dorsal openings (c, $\mathbf{h}$, dotted line) are detected. $\mathbf{f}$, i In stronger cuticle phenotypes cuticle remnants also show inverted appendages. A1 abdominal segment 1; T1 thoracic segment 1; scale bar $100 \mu \mathrm{m}$; all panels in all pictures: anterior to the left 


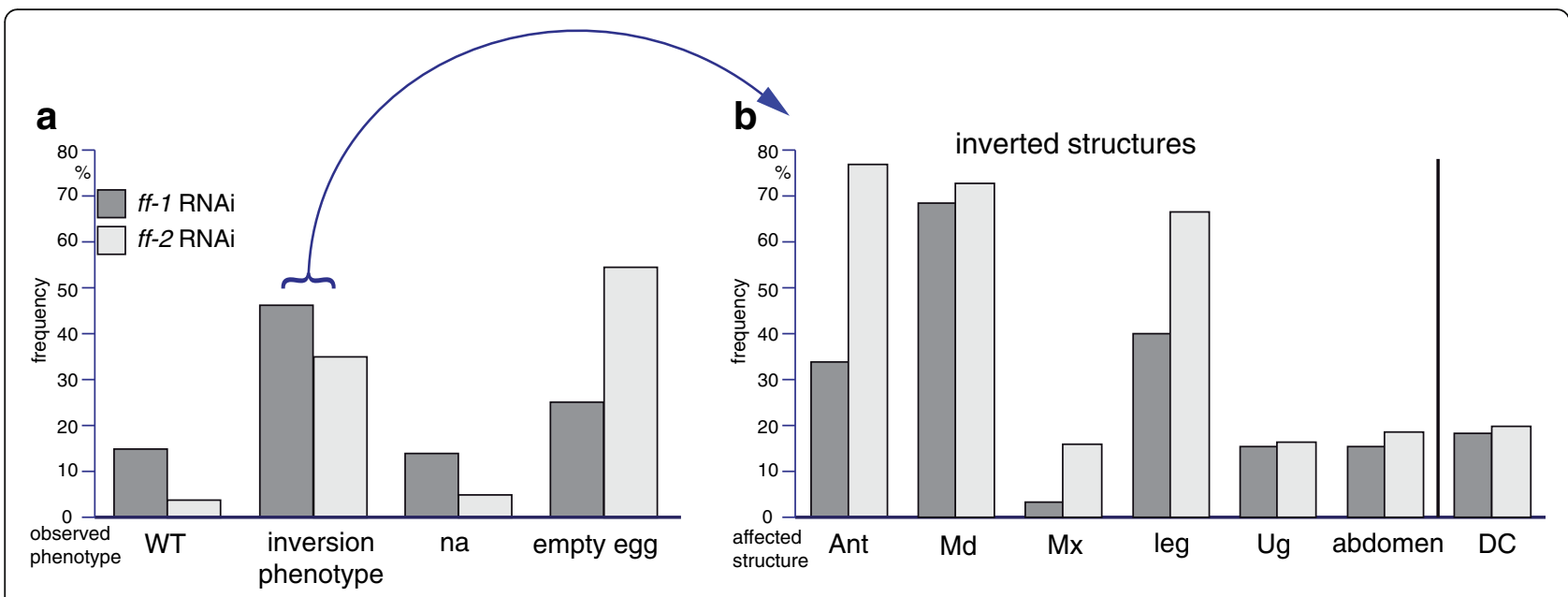

Fig. 4 Quantitative analysis of Tc-ff1 and Tc-ff2 phenotypes a Classification of cuticle phenotypes into wildtype-like (WT), analysable cuticles with inversions, not analysable cuticles (na) and "empty eggs" without visible cuticle within the vitelline membrane. b Detailed description of the "Analysable cuticles"-class (Arrow from A to B) subdivided into inversion events of antenna (Ant), mandible (Md), maxilla (Mx), leg, urogomphi (Ug) and abdominal segments as well as cuticles with a dorsal opening (DC). Ant antenna; DC dorsal closure; Md mandible; Mx maxilla; na not analysable; Ug urogomphi; WT wildtype

drumstick (Tc-drm, odd-skipped family) [16] as a marker for proximal appendage border. We found that eversion defects occur already at the beginning of appendage bud formation (Fig. $5 f^{\prime}$ ), becoming clearly visible in elongated buds. When focusing on the ventral side of the germ band not all limb buds are visible (Fig. $5 \mathrm{~d}$, circles). The missing limbs can be detected, however, as invaginated appendages at a deeper focal plane (Fig. $5 \mathrm{~d}^{\prime}$, arrows). Appendage marker gene analyses for Tc-drm (Fig. 5a-b") and $T c-D l l$ (Fig. 5c-d') revealed that inverted appendages display wildtype expression patterns, confirming this phenotype is due to defects in growth directionality but not faulty pattern formation. As a result, embryonic inverted appendages displayed the wildtype-specific expression pattern of both, Tc-drm expression at the segmental base (Fig. $5 b^{c}$ ) and $T c-D l l$ expression in the distal region (Fig. 5d').

It has been proposed that epithelial morphogenesis can depend on an elevated level of cell death [32]. To reveal whether this may play a role in the directionality of appendage growth in Tribolium, we analysed Tc-ff RNAi $^{\text {R }}$ embryos using an antibody detecting Dcp1 [42]. However, the ingression of cells does not appear to be accompanied by an elevated level of cell death in the limb primordium or its immediate neighbourhood (Fig. 5f).

\section{Knockdown of the highly-conserved Tc-RhoGEF2 gene displays a Tc-flipflop-like RNAi phenotype}

To find additional putative interaction partners of Tc-ff, we searched the iBeetle database [10] for the characteristic Tc-ff-like outside-in RNAi phenotype. This approach follows the logic that a similar phenotype indicates the involvement in a similar pathway. Indeed, appendage eversion was disrupted when a gene coding for a RhoGTP exchange factor was knocked down via RNAi. The analysis of the genomic region within the Tribolium genome revealed a gene model (au2.g3948.t2) that combines two adjacent models from previous annotations (TC003069 and TC003070). BLAST analysis identifies this gene as the Drosophila homolog of the highlyconserved Rho Guanine nucleotide Exchange Factor 2 in Tribolium (Tc-RhoGEF2). The lack of sequence conservation of the $T c-f f$ orphan genes made it difficult to relate their function to known cellular pathways. Fortunately, after the discovery of Tc-ffl, another outside-in leg phenotype was found during an investigation of lethal mutants identified in the GEKU transposon mutagenesis screen [55]: the insertion line KT221 produced homozygous embryos which occationally display outside-in appendages. The KT221 insertion is located in an intron of the Tc-RhoGEF2 gene identified via the iBeetle database. RNAi mediated knockdown of Tc-RhoGEF2 generally results in much more severely affected larval cuticles that often are hard to analyse. However, $T c-R h o G E F 2^{\mathrm{RNAi}}$ cuticles $(N=138)$ do reveal the characteristic Tc-ff-like phenotype of inverted appendages (29\%) and incomplete dorsal closure (58\%) (Fig. 6).

\section{Tc-flipflop and Tc-RhoGEF2 influence morphogenetic dynamics and polarity of extraembryonic membranes and embryonic tissues}

To determine the function of Tc-ff and Tc-RhoGEF2 in early embryogenesis we analysed RNAi embryos both via time-lapse microscopy and in fixed stages. We found that RNAi mediated knockdown of Tc-ff as well as Tc-RhoGEF2 influences the integrity of extraembryonic membranes and 

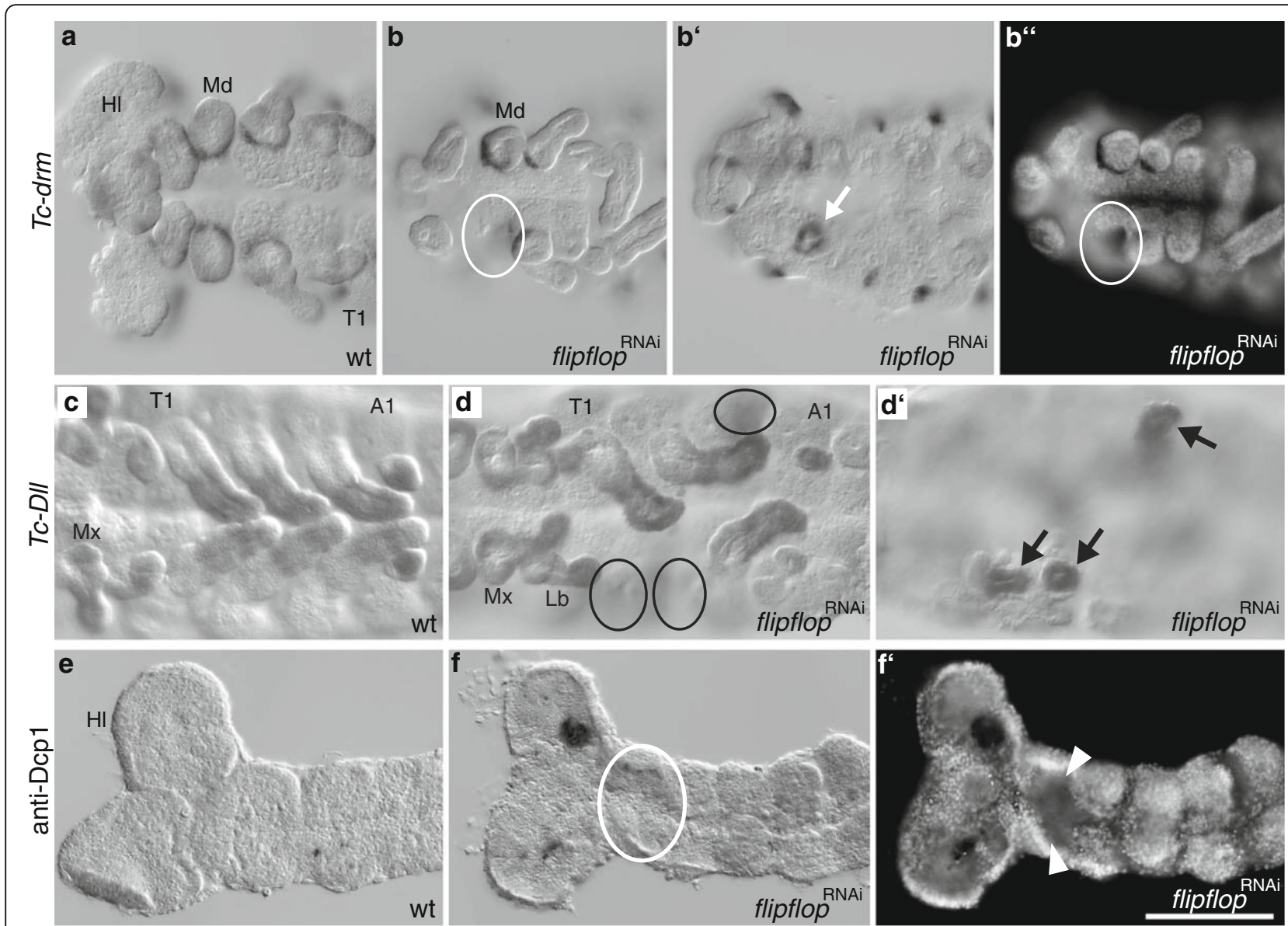

Fig. 5 Marker gene expression and apoptosis analysis in Tc-ff ${ }^{\text {RNAi }}$ embryos. Ventral focal plane: $a, b, b^{\prime \prime}, b, d$, e-f'. dorsal focal plane: $b^{\prime}, d^{\prime}$. a-b": Tc-drumstick; c-d': Tc-Distalless; e-f': apoptosis-marker anti-Dcp1. (a, b-b") Tc-drumstick (Tc-drm; TC006347) marker gene expression in embryonic wildtype (WT) and Tc-ff ${ }^{\text {NAi }}$ embryos marks the segmental base of appendages. (b, b') Inverted mandible clearly displays wildtype $T c-d r m$ expression at

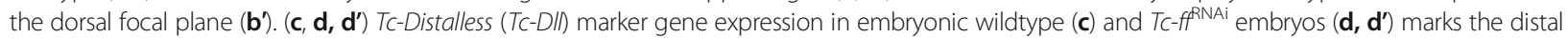
portion of each appendage except for the mandible. (d) Some leg anlagen are not visible in the ventral focal plane (circles) but are visible as inverted limb buds in the dorsal focal plane (arrows) (d'). (e-f) Apoptosis marker anti-Dcp1 in wildtype (e) and Tc-ff $f^{\text {RNAi }}$ embryos (f-f'). (d) Inverted appendage buds do not show elevated cell death at the stage of bud formation (circles). (f') Nuclear staining (DAPI) visualises embryonic morphology of inverted appendages after Tc-ff knockdown. A1 abdominal segment 1; Lb labium; Md mandible; Mx maxilla; T1 thoracic segment 1; scale bar 100 um; all panels in all pictures: anterior to the left
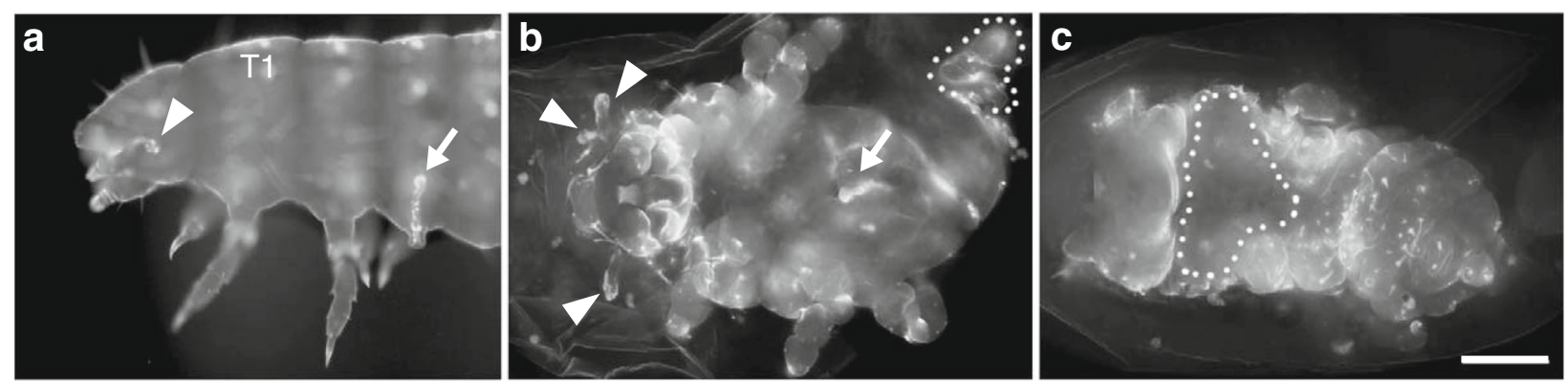

Fig. 6 Larval TC-RhoGEF2 ${ }^{\text {RNAi }}$ phenotype. a Magnified view of the KT221 mutant phenotype displaying an inverted leg (arrow) and head

appendage (arrowhead). b, c RhoGEF2 ${ }^{\text {RNAi }}$ cuticles display more severe defects: in addition to inverted appendages of the head (arrowheads) and thorax (arrows) RhoGEF2 knockdown also results in segmentation defects, a malformed posterior abdomen (b dotted outline) and dorsal closure defects (c, outlined with dots). Extended focus in all pictures combines several optical sections. a lateral view; b ventral view; c dorsal view); b, c scale bar $100 \mu \mathrm{m}$ 
interferes with morphogenetic movements of the young germ anlage and the germ band.

In late blastoderm stages we observed deep constrictions between the extraembryonic membrane and the site of the presumptive embryonic cells (Fig. 7b). Other embryos were able to form a germ band that is excluded from the serosa and displays a thickened posterior growth zone (Fig. 7d). Moreover, we frequently observed that germband extension underwent an S-shaped progression through the yolk (Fig. 7e). Such embryos often display holes along the ventral midline during older stages (Fig. 7g, h).

Via a live-imaging approach using a nGFP line [43] we observed that the extraembryonic membranes are functionally impaired. In the embryo shown in Fig. 8, the rupture and successive retraction of extraembryonic tissue during germ band elongation is visible while embryonic tissue develops holes and partly ingresses into the yolk.

\section{Discussion}

Genes that are required for the allocation and patterning of appendages are well-known [2, 59]. However, not much is known about the factors that determine the initial direction of tissue evagination during appendage bud formation. Here, we show that the novel genes Tc-ff1 and $T c-f f 2$ are required for early appendage eversion during embryogenesis of Tribolium. Knockdown of those genes leads to an outside-in phenotype of inverted appendages that has not been described so far. We further observed this highly specific appendage inversion phenotype after knockdown of RhoGEF2 function. This leads us to the hypothesis that the $T c-f f$ genes serve as important co-regulators within a Rho-dependent pathway.
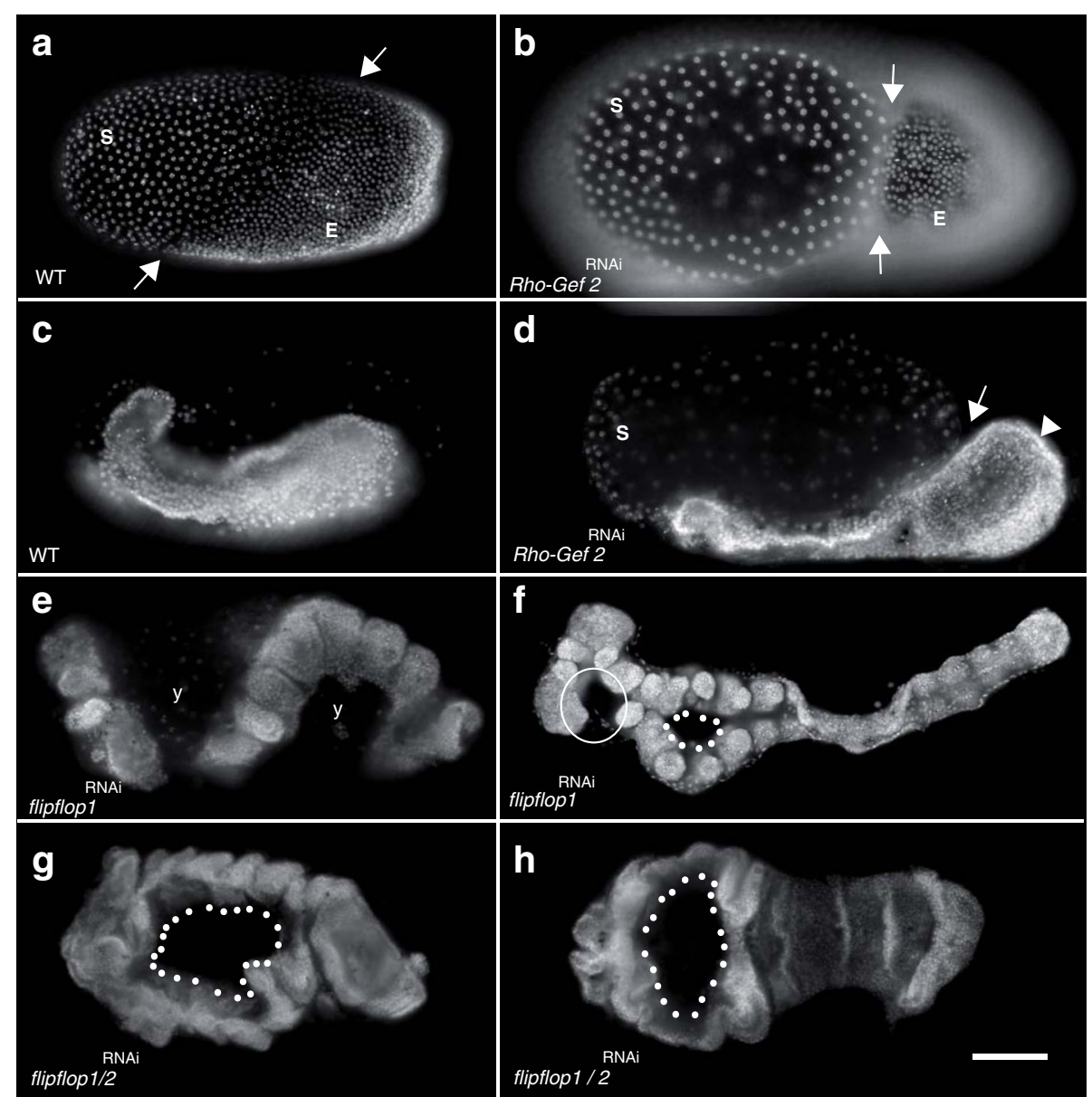

Fig. 7 flipflop- and RhOGEF2-RNAi knockdown interferes with early embryonic morphogenesis. a Wildtype blastoderm stage displays distinction between anterior extraembryonic tissue-anlagen (serosa S) and the posterior positioned embryonic cells (E); arrows mark the border. b Fixed RNAi embryos reveal tissue constrictions in blastoderm stages during embryonic anlagen formation (arrows). c Wildtype embryo prior to axis elongation. d Young RhoGEF2 ${ }^{\text {RNAi }}$ embryo with thickened posterior growth zone (arrowhead). The affected embryo is excluded from the serosa (arrow). $\mathbf{e}, \mathbf{h}$ Extended germband displays an unusual S-like orientation within the yolk (y). $\mathbf{e}$, inverted head appendages (f, circle) appear missing when viewed

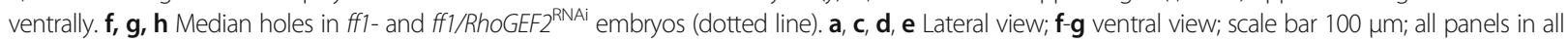
pictures: anterior to the left; Nuclear DAPI staining 


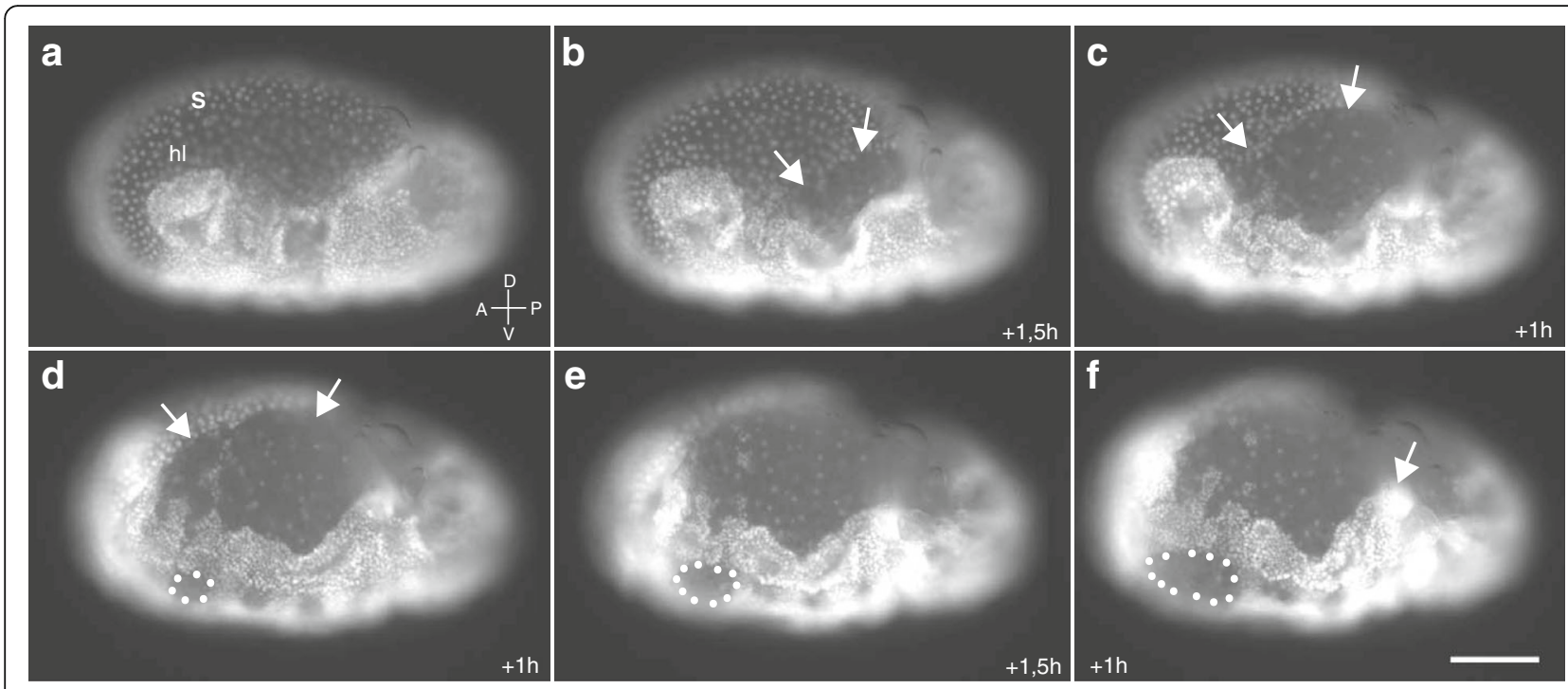

Fig. 8 Live imaging stills of an Tc-ff/RhoGEF2 double-RNAi embryo. a-f Developmental dynamics in a timeframe of 6 h, starting a few hours after egg lay. a Germ anlage covered by the intact serosa (S). b-f Rupture and successive retraction of extraembryonic membranes (arrows). $\mathbf{d}$-e Holes form in the median regions of the embryo (dotted outline) and ingression of embryonic tissue into the yolk (arrow in f). a-f Lateral view; anterior to the left, ventral down; scale bar $100 \mu \mathrm{m}$

\section{Eversion of embryonic limb anlagen requires the novel flipflop genes in Tribolium}

In the wildtype Tribolium embryo, limb development starts as a bud that everts and subsequently grows in length. For the first time, we identified genetic components required for this important initial decision within the limb field tissue. Initially, $T c-f f 1$ was uncovered via its enigmatic inverted leg phenotype during the iBeetle RNAi pre-screen using randomly picked cDNA clones as a source for dsRNA [48]. By sequence homology we identified a second flipflop gene in the Tribolium genome, named Tc-ff2. Both genes are predicted to code for small proteins of 136 and 127 amino acids, respectively, lacking any known functional domains. A web-tool based analysis of their sequences [1, 26] characterises the $T c-f f$ genes, at least in the absence of a binding partner, as "unstructured" with long stretches of low complexity domains.

To answer the question, whether the Tc-ff genes are indeed protein-coding or function as long-non-coding RNAs, we raised anti-peptide-antibodies that recognise a single specific band of the expected molecular weight (Additional file 1: Fig. S1A) in a Western blot using embryonic extracts. We take this as indication that the predicted ORFs are indeed translated, however, the antibody does not detect a distinct spatial or subcellular pattern in the embryo.

The $T c$-ff genes can be found in the Tribolium lineage (including T. confusum, T. madens and T. freemani. UCSC Genome Browser) but seem absent from any other sequenced genomes. Based on their lack of conservation, small size and apparent disordered structure, we classify $T c$-ff1 and Tc-ff2 as orphan genes [53]. Given that orphan genes of small size tend to be evolutionarily more recent [31], these genes may well be limited to a small subset of colepteran species. However, we also cannot exclude that the Tc-ff genes provide a conserved function and homologs cannot be identified due to small size and a fastevolving sequence. In any case, the $T c$-ff genes represent one of few examples of orphan genes with an essential early embryonic function. In addition, these genes illuminate an early decision in the development of appendages which had been overlooked so far.

With the Tc-ff knockdown we observed a variety of phenotypes in affected larval cuticles ranging from severe to weak. We categorise the inverted growth of one or just a few appendages without additional defects as a weak knockdown phenotype. More severely affected cuticles display many outside-in events at once combined with a dorsal closure defect (Fig. 3h). The strongest effects are represented by cuticle remnants without recognisable structures or the complete lack of a cuticle ("empty eggs"), respectively, underlining that Tc-ff function is required in different tissues. While the $T c-f f 2$ knockdown results in a somewhat higher penetrance regarding phenotypic defects compared to Tc-ff1, both genes appear to be non-redundant as the single knockdown of either gene is sufficient to disrupt their morphogenetic function. This is also highlighted by the fact that the combined Tc-ff1/ff2 double RNAi knockdown does not increase the overall penetrance compared to the single knockdowns (Additional file 2: Table S1). 
We found that the direction of limb budding in Tribolium is determined as early as the beginning of appendage bud formation and is significantly influenced by $T c-f f$ function. We have no indications that in Tribolium the eversion of the limb epithelium depends on an elevated level of cell death as it has been shown for other cases [32, 44].

\section{The flipflop genes may act in a RhoGEF-dependent cell polarity network}

Given that the orphan Tc-ff genes lack evolutionarily conserved characteristics to associate them with any known molecular pathway, we searched for other genes that display the same or a similar RNAi knockdown phenotype. We found that RNAi-mediated (partial) knockdown of the ubiquitously expressed Tribolium homolog of RhoGEF2 did also result in the disruption of appendage eversion, resembling the characteristic $T c-f f$ RNAi outside-in phenotype (Fig. 6). In contrast to Tc-ff, Tc-RhoGEF2 represents an evolutionarily highly conserved gene. By activating members of the Rho-family GTPase [3], RhoGEF2 plays an essential role in a number of morphological processes involving cell-cell adhesion, cell polarity, cell migration and cell motility [20, 36, 47]. Furthermore, RhoGEF2 is well known to be a key factor controlling cell shape change and apical constriction through the regulation of actomyosin contractility [3, 15, 24, 38]. In Drosophila an impairment of factors involved in Rho-dependent apical constriction can result in salivary gland formation outside the embryo, instead of forming inside the embryo as in wildtype [7]. Here, we show that both Tc-ff and Tc-RhoGEF2 initially determine the direction of appendage growth. However, the number and localisation of inside-out events seen in different $T c-f f^{\mathrm{RNAi}}$ larvae does not follow a certain pattern. This variability of the phenotype led us to hypothesise that the cell fate decision of the epithelium whether to invaginate or evaginate may be a quantitative local event rather than an all-or-nothing epithelial switch. Depending on the number of cells within the limb field undergoing uniform polarised constriction either at the apical or the basal side provides the physical ground for an epithelium to buckle to one of the two directions. Disruption of the uniformity of this collective cell behaviour through absence of the same polarity cue in all cells increases the likelihood of adjacent cells being forced into a different direction. In Drosophila it has been shown that inhibition of apical constriction in a defined area of the epithelial tissue disrupts ventral furrow formation depending on the number of cells affected as well as the intensity of the inhibitory signal [13]. Tissue invagination does still function when a smaller section of cells is affected as long as there are still enough cells undergoing cell shape change "dragging" adjacent corrupted cells with them.
Based on the findings in Drosophila and the highly conserved RhoGEF2 function, we hypothesise that in Tribolium ff and RhoGEF2 play an essential role in the decision where cellular constriction - either at the apical or basal side - takes place, so that in the absence of this apical-basal polarity cue the buckling direction becomes random. However, the genes are not required for the formation of the appendage primordium itself. Thus, we propose that $T c-f f$ is involved in a Rho GTPasedependent pathway that regulates the apico-basal polarity of a cell. However, a detailed analysis of the cellular dynamics including suitable markers localising proteins that are involved in cell shape change events will be required to validate our hypothesis.

\section{Tc-flipflop and Tc-RhoGEF2 are also required for the morphogenetic dynamics and polarity of extraembryonic membranes}

In contrast to the single reduced amnioserosa of Drosophila, Tribolium has two extraembryonic membranes, amnion and serosa, that actively contribute to the morphogenesis of Tribolium during gastrulation, germ band extension and dorsal closure [18, 39]. We have seen that knockdown of either Tc-ff or Tc-RhoGEF2 affects the directed morphogenetic movements and the cellular integrity of extraembryonic tissues. This aspect of Tc-ff and Tc-RhoGEF2 function is seen in the early embryo where the extraembryonic membranes fold prematurely and fail to fully cover the embryo. As a consequence, a misshaped germband forms on top of the yolk (Fig. 7d). This phenotype clearly illustrates that the dorso-posterior translocation of the extraembryonic tissues and the enwrapping of the embryo require coordinated tissue elongation within a plane of cells, a process likely to involve planar cell polarity (PCP) [57]. As described for other systems, Rho-dependent cell shape changes can be regulated through the PCP pathway that involves non-canonical Wnt signalling. Thus, we propose that the polarised maintenance of extraembryonic tissue dynamics during Tribolium embryogenesis depends on PCP and that Tc-RhoGEF2 and the Tc-ff genes are downstream targets of this important signalling pathway in Tribolium.

Additionally, we have observed premature ruptures of extraembryonic membranes in RNAi embryos (Fig. 8) as well as dorsal closure defects. In Drosophila, dorsal closure depends on actomyosin contractility at the apical cortex of the amnioserosa cells while the actin cable in cells of the leading edge seems dispensable for this process $[11,40]$. It is conceivable that the polarity of the extraembryonic cells along the apico-basal axis might also be disturbed in $T c-f f^{\mathrm{RNAi}}$ embryos and, as a consequence, dorsal closure is impaired. An unstructured actomyosin network also may contribute to weaken the integrity of 
the extraembryonic membranes epithelia so that they cannot withstand strong mechanical tension during the dorsal closure process. Once cell polarity markers become available for Tribolium it will be feasible to evaluate the involvement of PCP in the process of dorsal closure.

Based on our observations we hypothesise that $T c-f f$ and Tc-RhoGEF2 contribute to the polarisation of cell movement early in development but do not influence the differentiation of epithelial structures per se. Later, they are involved in stabilising the cellular components required for the pulling forces of tissues during dorsal closure.

Assuming that the Tc-ff genes act in the same pathways as Tc-RhoGEF2, we propose that they may be downstream targets of non-canonical Wnt/PCP signalling via the regulation of or in parallel to Rho proteins.

\section{Conclusions}

Here, we showed Tc-ff as one of the few examples of an orphan gene playing a crucial role in a developmental process such as in morphogenetic cell movements. This is one more example for an additional novel, species-specific or fast evolving factor that functions in an otherwise conserved pathway. Previously, an orphan gene within the BMP-pathway involved in digit formation and -outgrowth in the limb has been described in a vertebrate [23]. It will be interesting to see if functional equivalents of the Tc-ff orphan genes will be found in other organisms.

\section{Additional files}

Additional file 1: Figure S1. Flipflop expression analysis. (A) Western blots mark specific bands presumably representing the Flipflop proteins according to the expected molecular weight (FF1 14,47 kD; FF2 13,63 kD, asterisks). Unspecific bands of higher molecular weight may detect yolk proteins. (B-E) whole mount in situ hybridisation using Tc-flipflop1 and TC-flipflop2 mRNA antisense-probes displays ubiquitous expression patterns exemplarily shown in wildtype (wt) embryos both before and at the stage of bud formation. (F-I') Transcript detection in wildtype embryos $\left(\mathbf{F}, \mathbf{F}^{\prime}, \mathbf{H}, \mathbf{H}^{\prime}\right)$ and after knockdown of the respective gene $\left(\mathbf{G}, \mathbf{G}^{\prime}, \mathbf{I}, \mathbf{I}^{\prime}\right)$ to validate knockdown efficiency. (F', G', H', I') DAPI staining. Scale bar $100 \mu \mathrm{m}$; all panels in all pictures: anterior to the left. Western blot analysis. (PDF $150 \mathrm{~kb}$ )

Additional file 2: Table S1. Quantitative analysis of phenotypes. Table S2. Count of analysable inversion events per cuticle. Table S3. Quantitative analysis of the wildtype and "empty egg" phenotype. (PDF $32 \mathrm{~kb}$ )

\footnotetext{
Acknowledgements

We thank the following people for their help: Andrej Lupas for discussing with us the Flipflop protein structure; Verena Hofer-Pretz and Nicole Gehring for excellent technical assistance and beetle care. Lizzy Ge-rischer and Mario Stanke for providing Tribolium genome annotation tools. Christian Schmitt-Engel for the initial discovery of flipflop 1, and Jianwei Li for additional flipflop sequence information.
}

Funding

German Research Community DFG; Grant to AB (BE 4850/1-1).

\section{Authors' contributions}

MK: initial identification of the Tc-ff and KT221 phenotypes; ST, AB performing experiments; $S T, A B, R S$ initiation and conception of the experiments; ST, RS writing the manuscript; AB, MK corrections. All authors read and approved the final manuscript.

Ethics approval and consent to participate

Not applicable

Consent for publication

Not applicable

\section{Competing interests}

The authors declare that they have no competing interests.

\section{Publisher's Note}

Springer Nature remains neutral with regard to jurisdictional claims in published maps and institutional affiliations.

\section{Author details}

${ }^{1}$ Institut für Biowissenschaften, Universität Rostock, Albert-Einsteinstr 3, D-18059 Rostock, Germany. ${ }^{2}$ Universität Tübingen, Auf der Morgenstelle 15, D-72076 Tübingen, Germany. ${ }^{3}$ Friedrich-Alexander-Universität Erlangen-Nürnberg, Department Biologie Abt. Entwicklungsbiologie, Staudtstr. 5, D-91058 Erlangen, Germany.

Received: 12 June 2017 Accepted: 3 October 2017

Published online: 19 October 2017

\section{References}

1. Alva V, Nam SZ, Söding J, Lupas AN. The MPI bioinformatics toolkit as an integrative platform for advanced protein sequence and structure analysis. Nucleic Acids Res. 2016:44:W410-5.

2. Angelini DR, Kaufman TC. Insect appendages and comparative ontogenetics. Dev Biol. 2005;286:57-77.

3. Barrett K, Leptin M, Settleman J. The Rho GTPase and a putative RhoGEF mediate a signaling pathway for the cell shape changes in Drosophila gastrulation. Cell. 1997;91:905-15.

4. Beermann A, Jay DG, Beeman RW, Hülskamp M, Tautz D, Jürgens G. The Short antennae gene of Tribolium is required for limb development and encodes the orthologue of the Drosophila Distal-less protein. Development. 2001;128:287-97.

5. Brown, SJ, Shippy, TD, Miller, S, Bolognesi, R, Beeman, RW, Lorenzen, MD, Bucher, G, Wimmer, EA \& Klingler, M. The Red Flour Beetle, Tribolium castaneum (Coleoptera): A Model for Studies of Development and Pest Biology. Cold Spring Harb Protoc 2009, doi:https://doi.org/10.1101/pdb. emo126.

6. Cadigan KM, Nusse R. Wnt signaling: a common theme in animal development. Genes Dev. 1997;11:3286-305.

7. Chung, S, Kim, S \& Andrew, DJ. Uncoupling apical constriction from tissue invagination. Elife. 2017;6:e22235,

8. Cohen SM. Specification of limb development in the Drosophila embryo by positional cues from segmentation genes. Nature. 1990;343:173-7.

9. Dong B, Deng W, Jiang D. Distinct cytoskeleton populations and extensive crosstalk control Ciona notochord tubulogenesis. Development. 2011;138: 1631-41.

10. Dönitz J, Schmitt-Engel C, Grossmann D, Gerischer L, Tech M, Schoppmeier M, Klingler M, Bucher G. iBeetle-base: a database for RNAi phenotypes in the red flour beetle Tribolium castaneum. Nucleic Acids Res. 2015:43:D720-5.

11. Ducuing A, Vincent $\mathrm{S}$. The actin cable is dispensable in directing dorsal closure dynamics but neutralizes mechanical stress to prevent scarring in the Drosophila embryo. Nat Cell Biol. 2016;18:1149-60.

12. Graf L, Gierer A. Size, shape and orientation of cells in budding Hydra and regulation of regeneration in cell aggregates. Wilhelm Roux Arch Dev Biol. 1980:188:141-51.

13. Guglielmi G, Barry JD, Huber W, De Renzis S. An optogenetic method to modulate cell contractility during tissue morphogenesis. Dev Cell. 2015;35:646-60.

14. Gutzman JH, Graeden EG, Lowery LA, Holley HS, Sive H. Formation of the zebrafish midbrain-hindbrain boundary constriction requires laminindependent basal constriction. Mech Dev. 2008;125:974-83.

15. Häcker U, Perrimon N. DRhoGEF2 encodes a member of the Dbl family of oncogenes and controls cell shape changes during gastrulation in Drosophila. Genes Dev. 1998;12:274-84. 
16. Hao I, Green RB, Dunaevsky O, Lengyel JA, Rauskolb C. The odd-skipped family of zinc finger genes promotes Drosophila leg segmentation. Dev Biol. 2003;263:282-95.

17. Harden N. Signaling pathways directing the movement and fusion of epithelial sheets: lessons from dorsal closure in Drosophila. Differentiation. 2002;70:181-203.

18. Hilbrant, M, Horn, T, Koelzer, S \& Panfilio, KA. The beetle amnion and serosa functionally interact as apposed epithelia. Elife. 2016;5: e13834.

19. Hopyan S, Sharpe J, Yang Y. Budding behaviors: growth of the limb as a model of morphogenesis. Dev Dyn. 2011;240:1054-62.

20. Kaibuchi K, Kuroda S, Fukata M, Nakagawa M. Regulation of cadherin-mediated cell-cell adhesion by the Rho family GTPases. Curr Opin Cell Biol. 1999;11:591-6.

21. Kerridge S, Munjal A, Philippe JM, Jha A, de las Bayonas AG, Saurin AJ, Lecuit T. Modular activation of Rho1 by GPCR signalling imparts polarized myosin II activation during morphogenesis. Nat Cell Biol. 2016;18:261-70.

22. Kondo T, Hayashi S. Mechanisms of cell height changes that mediate epithelial invagination. Dev Growth Differ. 2015;57:313-23.

23. Kumar A, Gates PB, Czarkwiani A, Brockes JP. An orphan gene is necessary for preaxial digit formation during salamander limb development. Nat Commun. 2015;6:8684

24. Lecuit T, Lenne PF. Cell surface mechanics and the control of cell shape, tissue patterns and morphogenesis. Nat Rev Mol Cell Biol. 2007;8:633-44.

25. Lee JY. Uncorking gastrulation: the morphogenetic movement of bottle cells. Wiley Interdiscip Rev Dev Biol. 2012;1:286-93.

26. Letunic I, Doerks T, Bork P. SMART: recent updates, new developments and status in 2015. Nucleic Acids Res. 2015;43:D257-60.

27. Martin AC, Goldstein B. Apical constriction: themes and variations on a cellular mechanism driving morphogenesis. Development. 2014;141:1987-98.

28. Martinez-Morales JR, Rembold M, Greger K, Simpson JC, Brown KE, Quiring R, Pepperkok R, Martin-Bermudo MD, Himmelbauer H, Wittbrodt J. ojoplanomediated basal constriction is essential for optic cup morphogenesis. Development. 2009;136:2165-75.

29. Martinez-Morales JR, Wittbrodt J. Shaping the vertebrate eye. Curr Opin Genet Dev. 2009;19:511-7.

30. Mayor R, Theveneau E. The role of the non-canonical Wnt-planar cell polarity pathway in neural crest migration. Biochem J. 2014;457:19-26.

31. McLysaght A, Hurst LD. Open questions in the study of de novo genes: what, how and why. Nat Rev Genet. 2016;17:567-78.

32. Monier B, Gettings M, Gay G, Mangeat T, Schott S, Guarner A, Suzanne M. Apico-basal forces exerted by apoptotic cells drive epithelium folding. Nature. 2015;518:245-8.

33. Nicolas-Perez, M, Kuchling, F, Letelier, J, Polvillo, R, Wittbrodt, J \& MartinezMorales, JR. Analysis of cellular behavior and cytoskeletal dynamics reveal a constriction mechanism driving optic cup morphogenesis. Elife. 2016;5:e15797

34. Nikolopoulou E, Galea GL, Rolo A, Greene ND, Copp AJ. Neural tube closure: cellular, molecular and biomechanical mechanisms. Development. 2017;144: 552-66

35. Nishimura T, Honda H, Takeichi M. Planar cell polarity links axes of spatial dynamics in neural-tube closure. Cell. 2012;149:1084-97.

36. Nobes C, Hall A. Regulation and function of the Rho subfamily of small GTPases. Curr Opin Genet Dev. 1994;4:77-81.

37. Nomachi A, Nishita M, Inaba D, Enomoto M, Hamasaki M, Minami $Y$. Receptor tyrosine kinase Ror2 mediates Wnt5a-induced polarized cell migration by activating c-Jun $\mathrm{N}$-terminal kinase via actin-binding protein filamin A. J Biol Chem. 2008:283:27973-81.

38. Padash Barmchi M, Rogers S, Häcker U. DRhoGEF2 regulates actin organization and contractility in the Drosophila blastoderm embryo. J Cell Biol. 2005;168:575-85.

39. Panfilio KA, Oberhofer G, Roth S. High plasticity in epithelial morphogenesis during insect dorsal closure. Biol Open. 2013;2:1108-18.

40. Pasakarnis L, Frei E, Caussinus E, Affolter M, Brunner D. Amnioserosa cell constriction but not epidermal actin cable tension autonomously drives dorsal closure. Nat Cell Biol. 2016;18:1161-72.

41. Rogers SL, Wiedemann U, Häcker U, Turck C, Vale RD. Drosophila RhoGEF2 associates with microtubule plus ends in an EB1-dependent manner. Curr Biol. 2004;14:1827-33.

42. Sarkissian T, Timmons A, Arya R, Abdelwahid E, White K. Detecting apoptosis in Drosophila tissues and cells. Methods. 2014;68:89-96.

43. Sarrazin AF, Peel AD, Averof M. A segmentation clock with two-segment periodicity in insects. Science. 2012;336:338-41.
44. Saunders JW Jr, Gasseling MT. Cellular death in morphogenesis of the avian wing. Dev Biol. 1962;5:147-78.

45. Sawyer JM, Harrell JR, Shemer G, Sullivan-Brown J, Roh-Johnson M, Goldstein B. Apical constriction: a cell shape change that can drive morphogenesis. Dev Biol. 2010;341:5-19.

46. Schinko, J, Posnien, N, Kittelmann, S, Koniszewski, N \& Bucher, G. Single and double whole-mount in situ hybridization in red flour beetle (Tribolium) embryos. Cold Spring Harb Protoc. 2009. pdb.prot5258.

47. Schlessinger $\mathrm{K}$, Hall A, Tolwinski N. Wnt signaling pathways meet Rho GTPases. Genes Dev. 2009;23:265-77.

48. Schmitt-Engel C, Schultheis D, Schwirz J, Strohlein N, Troelenberg N, Majumdar U, Dao VA, Grossmann D, Richter T, Tech M, et al. The iBeetle large-scale RNAi screen reveals gene functions for insect development and physiology. Nat Commun. 2015;6:7822.

49. Schröder R, Beermann A, Wittkopp N, Lutz R. From development to biodiversity-Tribolium castaneum, an insect model organism for short germband development. Dev Genes Evol. 2008;218:119-26.

50. Schultz J, Milpetz F, Bork P, Ponting CP. SMART, a simple modular architecture research tool: identification of signaling domains. Proc Natl Acad Sci U S A. 1998;95:5857-64.

51. Shippy, TD, Coleman, CM, Tomoyasu, Y \& Brown, SJ. Concurrent in situ hybridization and antibody staining in red flour beetle (Tribolium) embryos. Cold Spring Harb Protoc 2009, pdb.prot5257.

52. Söding J, Biegert A, Lupas AN. The HHpred interactive server for protein homology detection and structure prediction. Nucleic Acids Res. 2005;33:W244-8

53. Tautz D, Domazet-Lošo T. The evolutionary origin of orphan genes. Nat Rev Genet. 2011;12:692-702

54. Tickle, C. How the embryo makes a limb: determination, polarity and identity. J Anat. 2015; 4:418-30.

55. Trauner J, Schinko J, Lorenzen MD, Shippy TD, Wimmer EA, Beeman RW Klingler M, Bucher G, Brown SJ. Large-scale insertional mutagenesis of a coleopteran stored grain pest, the red flour beetle Tribolium castaneum, identifies embryonic lethal mutations and enhancer traps. BMC Biol. 2009;7:73.

56. Varner VD, Nelson CM. Cellular and physical mechanisms of branching morphogenesis. Development. 2014;141:2750-9.

57. Wallingford JB. Planar cell polarity and the developmental control of cell behavior in vertebrate embryos. Annu Rev Cell Dev Biol. 2012;28:627-53.

58. Webster G, Hamilton S. Budding in Hydra: the role of cell multiplication and cell movement in bud initiation. J Embryol Exp Morphol. 1972;27:301-16.

59. Wolpert L. Pattern formation in epithelial development: the vertebrate limb and feather bud spacing. Philos Trans R Soc Lond B Biol Sci. 1998;353:871-5.

60. Wyngaarden LA, Vogeli KM, Ciruna BG, Wells M, Hadjantonakis AK, Hopyan S. Oriented cell motility and division underlie early limb bud morphogenesis. Development. 2010;137:2551-8.

\section{Submit your next manuscript to BioMed Central and we will help you at every step:}

- We accept pre-submission inquiries

- Our selector tool helps you to find the most relevant journal

- We provide round the clock customer support

- Convenient online submission

- Thorough peer review

- Inclusion in PubMed and all major indexing services

- Maximum visibility for your research

Submit your manuscript at www.biomedcentral.com/submit
C) Biomed Central 